\title{
Exponential Technologies and the Perfect Storm for Digital Health
}

\author{
Vijay Chandru*
}

I was born in 1953, the year Mount Everest was scaled and the double helical structure of DNA was solved. Alan Turing and Claude Shannon had already made their incredible breakthroughs in information science and theory, while computing machines were just about making a mark in public consciousness. The UNIVAC computer had successfully predicted the outcome of the US presidential elections in 1952 on television. With a sample of just $1 \%$ of the voting population, it famously predicted an Eisenhower landslide. For those of us who have worked in technology for the last seven decades, we feel extraordinarily fortunate to have witnessed and benefitted from the parallel advances in computing and molecular biology over the years. By some reasonable calculations, we can argue that computing entered the second half of the chessboard, an acceleration by a factor of $2^{32}$ by 2006 some 48 years after the advent of Moore's Law. Genome or DNA sequencing was not far behind and also made the cut, about 10 years later.

It was no surprise that by 2012 that a top physician and genomics professor at the Scripps Translational Institute, Dr Eric Topol, was calling for medicine to be Schumpetered and called it a "Kairos moment in medicine." For Dr Topol and his followers, the intellectual excitement in medicine was that we were on the verge of digitizing humans. But as Nandan and Seethalakshmi have pointed out in the lead article of this issue, the call for this digital or radical change in medicine could also come from social crises-either the spiraling costs of healthcare in some societies or the crying need for access in others. The latter is also poignantly brought out in the recent treatise "Bridgital Nation: Solving technology's people problem" as reviewed by Professor Rudra Pratap in this issue.

As Professor Ananthasuresh points out in the editorial, the idea of starting initiatives in digital health has been a topic of deep discussion for a group of us in the faculty of the Indian Institute of Science for a few seasons now. The image on the front cover of this issue was designed by
Professor Rudra Pratap who has been steering the digital health initiative in the campus. Incidentally, the repeated binary code in the figure carries a relevant message that the reader is invited to decipher. The workshop on digital health held in the Winter of 2019 in some ways channeled our focus and a tidy design of this special issue took shape. Indeed, a few papers presented at the workshop have been included in this issue.

The perfect storm of COVID-19 struck soon after and the digital epidemiology elective that I had been offering at the Institute since 2017, in which we simulated nightmare pandemic scenarios in a classroom, were actually playing out as an unmitigated global human disaster. After the initial shock that we all experienced, I was delighted to see the enthusiasm and dedication with which our scientific communities locally and globally rose to the occasion. It seemed imperative that this response needed to be documented. With the support of the Editor-in-chief, the Journal Office, our publishers and many academics and researchers who raced to document their insights and research on how digital can help, this issue has literally doubled in scope and size. I am really grateful to all of them for having afforded me this opportunity to chaperone this perfect storm for digital health.

We have organized the articles and communications in five sections. In the first section, we have a range of issues in health policy and practice prompted by the COVID-19 pandemic. Shahid Jameel reminds us of the global nature of biological threats that pandemics represent and why we need to connect the dots of sustainable development if we intend to survive them. Rahul Matthan takes us through a socio-legal analysis and narrative on the issue of data privacy and the compulsions of managing a pandemic. This is followed by the paper describing "Go Corona Go", where Yogesh Simmhan, Tarun Rambha et al., of IISc describe the institutional contact tracing and privacy preserving app that they have developed, and the conscious and sometime contrarian design choices they have made. The section is
${ }^{1}$ Indian Institute of Science, Bangalore, India.

*vijaychandru@iisc.ac.in 
concluded with a delightful vignette communication that describes the COVID Study Circle-a journal of a digital collective of research scientists, public policy wonks, physicians from the front lines and epidemiologists who met twice a week for 3 months to compile data, read, share information and synthesize, analyze and reflect. "The COVID Study Circle" often felt like the pandemic analogue of a clinical tumor board perplexed by the complexity of the wicked COVID-19.

The second section of papers in computational and systems biology begins with a review of a major human organ digital twin project-the virtual liver. This ambitious project was carried out at Strand Life Sciences, a spin off company from the Indian Institute of Science and the narrator, Kas Subramanian, was the chief scientist who led the project. RxCovea a global digital collective with the goal of curing COVID for ever and for all, steered by Bud Mishra, ponders the pandemic and builds a game theoretic host-pathogen model and explores its implications. And then the paper, by Sahoo, Jhunjhunwla and Jolly, describes the interplay between the immune system and virus and the therapies.

Health informatics and knowledge engineering forms the theme of the next section of five papers. We begin with a succinct but truly informative piece by Sameer Bhat, the founder of eClinicalWorks, who takes us through international anecdotes that show how electronic health records can have valuable impact on population health and wellness effected through preventive strategies. One of the promises of digital in preventive care in rural India and particularly in chronic non-communicable diseases (NCDs) has been an enduring theme of many social health initiatives. The paper by Sunita Nadhamuni et al., describes a public private partnership between Dell Technologies and the National Health Systems Resource Center which has successfully built and rolled-out healthcare technology at scale to reach millions of people across India. Population health data when rendered geospatially can be insightful and a foundation for analysis. The Health Heatmap of India project described in the paper by Akshay Dinesh et al., also argues the case for an open data framework so that the heatmaps created becomes a public good that researchers can add modeling and analysis layers to. The short communication by Pandit and Vanak that follows, suggests how in the absence of strong data one can still approach causal analysis of 'one health' propositions using knowledge engineering methods based on inductive reasoning, a classical methodology in artificial intelligence. The final paper by Collard et al., in this section is another exemplar in the use of knowledge engineering that describes how the root and rule-based analysis was used to create a very useful web resource CORD-19 (COVID-19 Open Research Dataset) at NIST (National Institute of Standards and Technology).

We noted earlier, the extraordinary acceleration in genomics that we have witnessed particularly in the new millennium. By 2010, the whole genome sequencing of a 5-yearold boy Nicholas Volcker led to a sharp diagnosis of an immune deficiency and the choice of a transplantation therapy that saved him and cured him. The story captured by the Milwaukee Sentinel in the Pulitzer prize winning journalistic series "One in a Billion: a boy's life, a medical mystery" launched personalized medicine which gradually morphed into precision medicine. We have four papers in the section on precision health technologies. The first takes us through the sophistication needed in analyzing genomic data of rare genetic diseases like Nic Volcker's and how powerful data analysis tools built by bioinformaticians like Vamsi Veeramachaneni have now made it possible to provide such diagnostic services as a standard of care. The papers by Ram Sriram and Subramanian and by Satya Dash take us through the whole slew of digital technologies that have made precision health at population scale a reality. The fourth paper by Gopalkrishnan and Krishna describe a very clever boost in efficiency that they brought to SARS-Cov2 testing using a compressed sensing method called Tapestry Pooling which was described in the July 10th issue of Nature as a mathematical method that could transform Corona virus testing.

The sudden and savage nature of the ongoing COVID-19 pandemic has certainly caught everyone's attention. ${ }^{1}$ The fact that it has happened when the globe is so well connected thanks to information technology has made epidemiologists of just about anyone who has some mathematical ability and appreciation of infectious disease dynamics. However, there are some serious mathematicians and data scientists who had been interested in the power of computational epidemiology in counterfactual reasoning and in the predictive power of data driven models. There are also some very talented minds that threw themselves into the

\footnotetext{
${ }^{1}$ Viruses, Pandemics, and Immunity, A K Chakraborty and A Shaw, Illustrated by P J S Stork, MIT Press 2020.
} 
COVID challenge to try various ideas in pushing the state of the art. The prediction by the Global Virome Project ${ }^{2}$ that we could have around three zoonotic episodes a year that would have pandemic potential implies that we do need the best minds to help us prepare for the next one.

I must have heard about a hundred or so presentations and read preprints of many such ideas-and have picked a few of my favourites to present in the final section of this issue. To my delight all my top choices agreed to contribute papers and so we have a great collection here. To get us started we have a survey of mathematical models with a comparative analysis by Dubashi et al., a great primer for anyone getting started in the field. The idea of building a city scale agent-based simulator for counterfactual analysis that was actually used in Mumbai and Bangalore was a major multiinstitutional effort that is described by Rajesh Sundaresan et al. Bhattacharya and Vinay use small world networks and lattice models to propose normative strategies for suppressing the transmission of disease. Gopalan and Tyagi use models to address ground realities of testing and interventions in containment zones. The Stanford group of Vasudevan et al., have shared a communication that summarizes their findings in the disparities and variation in COVID-19 data reporting across India. The communication by Chakraborty and Chandru describes a gaussian process modeling approach for predicting vector borne disease incidence. The paper from the Virginia group of Adiga et al., points out that data-driven and mathematical modeling for response is going to be specific to the stages of a pandemic-pre-pandemic, acceleration, mitigation, suppression and post-pandemic (peace time). They have given the world a blueprint for getting prepared for the next one and the one after. We have learned to live with all kinds of disasters. Digital health will surely have its corner in disaster sciences, an emerging trans-discipline.

The digital revolution has not stalled and we are already some 14 years past the watershed of the second half of the chessboard with Moore's Law. The business pundits are calling this the cognitive era, the fourth industrial age and elucidation of every idea now uses "AI" or "Deep" as an adjective to be relevant to this trend. As always, the hype precedes reality. However, in 5 years, Eric Topol's third tome, "Deep medicine: how artificial intelligence can make healthcare human again" may well define the future of digital health. As the physician Abraham Verghese says in the foreword, "These historians of the now begin with the study of what is today, asking not how to avoid the perils of the past but how to maximize the advantages of the future."

Vijay Chandru

Guest Editor

\section{Publisher's Note}

Springer Nature remains neutral with regard to jurisdictional claims in published maps and institutional affiliations.

Published online: 1 October 2020

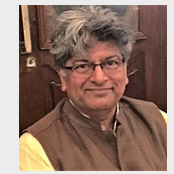

Vijay Chandru is computational mathematician by training and a fellow of the Indian Academy of Sciences and the Indian National Academy of Engineering. He is currently INAE Distinguished Technologist and an Adjunct Professor at the Indian Institute of Science (IISc) in Bangalore. He co-founded Strand Life Sciences, a spinoff of IISc in precision medicine, which he led as executive chairman from inception in 2000 till his retirement in 2018. He also co-founded several non-profit organizations-CHET, Metastring and OPFORD Foundations which are dedicated to health policy and outreach, the democratization of data and healthcare access for the underserved orphan diseases. A former president of ABLE, the biotechnology industry apex body of India, Professor Chandru is a technology pioneer of the World Economic Forum, where he has served on the industry advisory council on the future of health.

\footnotetext{
${ }^{2}$ https://www.globalviromeproject.org/.
} 